\title{
A Randomized Controlled Trial of an Internal Family Systems-based Psychotherapeutic Intervention on Outcomes in Rheumatoid Arthritis: A Proof-of-Concept Study
}

Nancy A. Shadick, Nancy F. Sowell, Michelle L. Frits, Suzanne M. Hoffman, Shelley A. Hartz, Fran D. Booth, Martha Sweezy, Patricia R. Rogers, Rina L. Dubin, Joan C. Atkinson, Amy L. Friedman, Fernando Augusto, Christine K. Iannaccone, Anne H. Fossel, Gillian Quinn, Jing Cui, Elena Losina, and Richard C. Schwartz

\begin{abstract}
Objective. To conduct a proof-of-concept randomized trial of an Internal Family Systems (IFS) psychotherapeutic intervention on rheumatoid arthritis (RA) disease activity and psychological status.

Methods. Patients with RA were randomized to either an IFS group for 9 months $(n=39)$ or an education (control) group $(n=40)$ that received mailed materials on RA symptoms and management. The groups were evaluated every 3 months until intervention end and 1 year later. Self-assessed joint pain (RA Disease Activity Index joint score), Short Form-12 physical function score, visual analog scale for overall pain and mental health status (Beck Depression Inventory, and State Trait Anxiety Inventory) were assessed. The 28-joint Disease Activity Score-C-reactive Protein 4 was determined by rheumatologists blinded to group assignment. Treatment effects were estimated by between-group differences, and mixed model repeated measures compared trends between study arms at 9 months and 1 year after intervention end.

Results. Of 79 participants randomized, 68 completed the study assessments and $82 \%$ of the IFS group completed the protocol. Posttreatment improvements favoring the IFS group occurred in overall pain [mean treatment effects -14.9 (29.1 SD); $\mathrm{p}=0.04$ ], and physical function [14.6 (25.3); $\mathrm{p}=0.04]$. Posttreatment improvements were sustained 1 year later in self-assessed joint pain $[-0.6$ (1.1); $\mathrm{p}=0.04]$, self-compassion [1.8 (2.8); $\mathrm{p}=0.01]$, and depressive symptoms [ $-3.2(5.0) ; \mathrm{p}=$ $0.01]$. There were no sustained improvements in anxiety, self-efficacy, or disease activity.

Conclusion. An IFS-based intervention is feasible and acceptable to patients with RA and may complement medical management of the disease. Future efficacy trials are warranted. Clinical Trials.gov identifier: NCT00869349. (First Release Aug 15 2013; J Rheumatol 2013;40:1831-41; doi:10.3899/jrheum.121465)
\end{abstract}

Key Indexing Terms: RHEUMATOID ARTHRITIS CLINICAL TRIALS

\author{
DISEASE ACTIVITY \\ PSYCHOTHERAPY
}

\section{DEPRESSION BEHAVIORAL MEDICINE}

Despite effective pharmacotherapy, many individuals with rheumatoid arthritis (RA) suffer ongoing pain and disability. Living with RA can lead to depression, anxiety, isolation, an overall impaired quality of $\operatorname{life}^{1,2}$, and
From the Department of Medicine, Division of Rheumatology, Immunology and Allergy, Brigham and Women's Hospital, Harvard Medical School; Department of Psychiatry, Cambridge Health Alliance, Boston, Massachusetts; Center for Self Leadership, Oak Park, Illinois; and Department of Psychology, Massachusetts School of Professional Psychology, Boston, Massachusetts, USA.

Supported by the Bristol Myers Squibb Foundation, ABBVIE, and Genentech.

N.A. Shadick, MD, MPH, Department of Medicine, Division of Rheumatology, Immunology and Allergy, Brigham and Women's Hospital, Harvard Medical School; N.F. Sowell, MSW, Department of Psychiatry, Cambridge Health Alliance; M.L. Frits, Department of Medicine, Division of Rheumatology, Immunology and Allergy, Brigham and Women's Hospital, Harvard Medical School; S.M. Hoffman, PhD; S.A. Hartz, RN, MSN, CS; F.D. Booth, MSW, Center for Self Leadership; M. Sweezy, PhD,
Department of Psychiatry, Cambridge Health Alliance;
P.R. Rogers, MSW; R.L. Dubin, Ed.D; J.C. Atkinson, MSW;
A.L. Friedman, MSW; F. Augusto, PhD, Center for Self Leadership;
C.K. Iannaccone, MPH; A.H. Fossel, Department of Medicine, Division of Rheumatology, Immunology and Allergy, Brigham and Women's Hospital, Harvard Medical School; G. Quinn, Department of Psychology, Massachusetts School of Professional Psychology; J. Cui, PhD; E. Losina, PhD, Department of Medicine, Division of Rheumatology, Immunology and Allergy, Brigham and Women's Hospital, Harvard Medical School; R.C. Schwartz, PhD, Center for Self Leadership.
Address correspondence to Dr. N.A. Shadick, Division of Rheumatology, Immunology and Allergy, Brigham and Women's Hospital, 75 Francis Street, Boston, MA 02115, USA. E-mail: nshadick@partners.org Full Release Article. For details see Reprints/Permissions at jrheum.org Accepted for publication June 25, 2013. 
increased healthcare resource use ${ }^{3}$. Psychotherapeutic interventions that improve disease activity, pain-related symptoms, and psychological function would be helpful to patients living with this disease.

A number of psychobehavioral interventions have been shown to be effective in improving coping efficacy and other outcomes in patients with $\mathrm{RA}^{4,5,6,7,8,9,10,11}$. Cognitive behavioral interventions, in particular, have reduced pain, joint inflammation, physical disability, and depression ${ }^{5,6,9,10}$. The improvements are variable according to the type of intervention, tend to be most effective in newly diagnosed patients, and have limited sustainability ${ }^{6,9}$. For example, effect sizes (ES) for pain and disability in 2 metaanalyses of psychological interventions for RA were modest ${ }^{12,13}$. Also, joint inflammation and swelling were reduced by several interventions, but these results were mostly seen in patients with illness of shorter duration ${ }^{13}$. In a Cochrane review assessing the effectiveness of educational programs for RA, there were positive effects on disability, joint counts, patient global assessments, psychological status, and depression, but the improvements were short-lived ${ }^{14}$. A sustainable intervention that affects disease activity in individuals with longer-term illness could improve patients' lives.

The Internal Family Systems (IFS) model is a rapidly emerging individual psychotherapeutic modality developed by Schwartz ${ }^{15}$ that teaches patients to attend to and interact with their internal experience mindfully. The model actively recruits self-compassion toward an individual's parts, conceptualized as subpersonalities that are manifested by strong feelings, judgments, or physical sensations. By fostering an internal dialogue with polarized thinking, IFS reduces emotional intensity and dysregulation; elements that have been shown to increase pain perception ${ }^{16}$ and disease activity in $\mathrm{RA}^{4,17}$. IFS also uses nonjudgmental noticing and active mindfulness. Mindfulness-based interventions have been helpful in a number of painful conditions including $\mathrm{RA}^{4,7,18}$. To date, more than 2200 therapists worldwide have been trained in the IFS modality ${ }^{19}$. This technique is increasingly being used as adjunctive therapy for certain medical conditions, with anecdotal benefit reported in migraines, back pain, and multiple sclerosis. To our knowledge, our study is the first to evaluate the IFS model's feasibility, acceptability, and potential efficacy in a randomized trial.

\section{MATERIALS AND METHODS}

Subjects. We conducted our study from September 2007 to February 2011 at the Brigham and Women's Hospital Arthritis Center in Boston. Adult subjects who were enrolled in the Brigham Rheumatoid Arthritis Sequential Study (BRASS) were eligible ${ }^{20}$ and were mailed a letter inviting them to participate in a 36-week intervention study. Subjects were screened by telephone, and if eligible, were invited to attend the baseline session. Inclusion criteria included a rheumatologist's diagnosis of RA, age $\geq 18$ years, not currently in an arthritis self-management program, and having active disease (they did not have to have stable disease, but they were excluded if they were in remission according to their rheumatologist). All subjects were under the medical care of their rheumatologist during the study.

Procedures. Subjects who agreed to participate signed an informed consent and did baseline assessments that were randomized to either the IFS program or an RA educational program of 36 weeks duration. Subjects were randomized by a computer algorithm and assigned by the project manager who had no direct patient contact. Allocation occurred with a fixed 1:1 allocation ratio of IFS intervention to control group after eligibility, consent, and baseline data were obtained. A sample size of 80 was set assuming about a $20 \%$ dropout rate, $\alpha$ error of 0.05 , and $80 \%$ power to detect an effect size of 0.70 on disease improvement, as found in O'Leary, et $a l^{11}$. Adverse events were monitored at each study visit and were reported to the Brigham and Women's Hospital Institutional Review Board according to the approved protocol. Study procedures and written informed consent were approved by the Institutional Review Board of the Brigham and Women's Hospital, Partners Health Care, Boston, Massachusetts.

Outcome measures. RA disease activity was evaluated by rheumatologists blinded to group assignment who assessed joint swelling and tenderness in 28 joints. All other outcomes were determined by subject self-report questionnaires. Assessments were made at baseline, 3-, 6-, and 9-month timepoints, and 1 year after the intervention's end. Questionnaires were filled out at baseline, 3, 6, 9, and 21 months by the subjects at the time of assessment, or mailed in if they were not completed during their visit. There was no study contact after 9 months until 1 year later.

The primary outcome measures were the improvement in disease activity, as measured by the Disease Activity Score-28-C-reactive Protein 4 DAS28-CRP4] and the RA Disease Activity Index (RADAI) joint score. The DAS28-CRP4 is a continuous measure reflecting the number of tender and swollen joints, patient global assessment of disease activity on a 100-mm visual analog scale (VAS), and the C-reactive protein (CRP) value. Determination of the CRP value was done using a validated high-sensitivity assay with a particle-enhanced immunoturbidimetric assay (Cobas C systems Roche/Hitachi). The RADAI joint score is a short patient questionnaire that assesses patient-reported disease activity by giving a summary of the total number of painful joints ${ }^{21,22}$

The Beck Depression Inventory (BDI), State Trait Anxiety Inventory (STAI), and Short Form-12 (SF-12) physical function scores were also assessed. Depression was measured using the $\mathrm{BDI}^{23}$, a validated and widely used scale for depression that contains 21 groups of statements that calculate the severity of depressive affect. Anxiety was measured using the validated Spielberger $\mathrm{STAI}^{24}$. It contains 20 items querying the respondent about how they feel at the time of response. Physical function health status was measured using the SF-12 physical function score ${ }^{25}$. A higher score indicated better function. Subjects were asked about their sleep quality using a $0-3$ scale; if over the past week they were able to get a good night's sleep without any difficulty (0), some difficulty (1), much difficulty (2), or unable to get a good night's sleep (3). The sleep quality measure is an item from the Multidimensional Health Assessment Questionnaire ${ }^{26}$, used in the clinic as a screen for problems with sleep; it has been shown to have good test-retest reliability and face validity. The sleep item is reported as a mean score. Participants were also given a 0-100 VAS to rate their pain ${ }^{26}$.

The secondary outcomes, self-efficacy and self-compassion, were measured. They included the Arthritis Self Efficacy Other Symptoms scale developed by Lorig, et $a l^{27}$ and the validated Self Compassion scale by Neff $^{28}$. The Neff Self Compassion scale is a 26-item self-report questionnaire, reported as a total self-compassion score that includes 6 factors: self-kindness, self-judgment, common humanity, isolation, mindfulness, and overidentification. It correlates with lower depression and anxiety outcomes and higher scores on a life satisfaction scale ${ }^{28}$. Its psychometric properties include good construct, content, and convergence validity, as well as good test-retest reliability, with a 26-item internal consistency score of $0.92^{28}$.

At each timepoint, we also collected information on arthritis medication usage. The medications were tabulated and then categorized into 
analgesics, nonsteroidal antiinflammatory drugs (NSAID), corticosteroids, nonbiological disease-modifying antirheumatic drugs (DMARD), and biologic therapies.

IFS intervention. Subjects attended group meetings that included 8 to 10 patients led by 1 trained IFS therapist who ran all of the groups throughout the study. Groups met every 2 weeks during the first 3 months and then monthly until study end. Additionally, subjects in the IFS intervention also participated in 15 biweekly, 50-min individual meetings over 36 weeks with one of the IFS-trained therapists.

The IFS protocol provided conceptual training and coaching in use of the IFS model. Participants were taught how to accept and understand their own feelings, such as fear, hopelessness, or anger in a compassionate way, as if they were members of their own internal family. They were led through exercises and participated in discussions designed to assist them in the identification of their individual emotional reactions to the pain and disability of RA, for example. All meetings were focused on RA symptoms and emotional states related to and exacerbating these symptoms. Once the patients learned the internal dialogue skills, they were encouraged to practice on their own at home. For example, noticing pain in a joint, identifying the accompanying emotions and cognitions, and approaching these internal states with interest and compassion, a patient would become more aware of the need to compromise between collapsing and stoically ignoring his or her pain. Plans could be altered to accommodate activities in a more reasonable, less extreme way. Additionally, the IFS intervention promoted an exploration of the origins of these emotional reactions and negative beliefs, allowing for changes and moderation in these extreme affects or cognitions ${ }^{15}$. Other topics that were included in the visits were fatigue and its attendant emotions, disability (either in 1 joint or overall), isolation related to the disability, and the effect of deformity. The overall purpose of the program was to enhance the subjects' skills in the use of the IFS model to reduce the disease burden, social isolation, and other behaviors exacerbating symptoms.

Intervention integrity. All the group sessions were run by 1 therapist (NFS). The 9 therapists who ran the individual sessions were trained in the study protocol before the program began, according to the study manual. The elements of and adherence to the IFS protocol were recorded in a study binder to document that the subjects were actively engaged in the treatment. All the therapists had received advanced training in IFS therapy and averaged 4 years of IFS therapy experience prior to the study. Four therapists were doctoral-level psychologists, 4 were social workers, and 1 was a certified nurse specialist in psychiatry. They had monthly meetings with 2 of the study's investigators (RCS, NFS) to ensure that the study protocol was maintained. The content of the group meetings is given in Table 1.

Table 1. Topics addressed in each session.

Session 1 Getting to know each other - "Where I am with my RA: Thinking in terms of different "parts' of my personality."

Session 2 My "Self" as my inner resource: the 8 C's

Session 3 RA and protective parts of my personality

Session 4 RA and vulnerable parts of my personality

Session 5 Can a relationship with my "Self" help my disease?

Session 6 What is scary about self-compassion when one lives with RA?

Session 7 Moving out of fear: the meaning of "Self" leadership

Session 8 Identifying inner conflict and bringing calm, compassion, and curiosity

Session 9 Listening to my body/listening to my parts: What's needed?

Session 10 Being with, not in, my emotional states: Speaking for, not from, my "parts"

Session 11 Vital needs, self-care, and my health

Session 12 Closure with each other and the way forward

RA: rheumatoid arthritis
$R A$ education group. The education group served as a minimal-attention control for the study. They met once as a group initially and then received monthly mailed educational information about RA, which was followed up by a phone call from a research assistant to reinforce the information. The mailed materials did not include any information on coping or stress reduction. The different topics delivered to the education group included (1) a group lecture on how exercise eases RA; (2) a recording of a lecture on DMARD; (3) information from an educational lecture on assistive devices for RA; (4) information on common laboratory tests and procedures for the diagnosis of RA; (5) information on diet and nutrition in RA; (6) information on osteoarthritis and how it differs from RA; (7) information about the immune system and its link to RA; and (8) completion of the program with review and education about the Brigham and Women's Hospital's ongoing patient seminar series and patient support programs.

Statistical analysis. Baseline comparisons between groups were compared using chi-square or t test analyses, as appropriate. Treatment effects were reported as mean between-group differences from baseline to the 9-month and 21-month followup visits, using $t$ tests to determine the differential effect of the intervention versus the education group. We adjusted for the DAS28-CRP4 and self-efficacy score differences in a general linear regression equation with baseline value and treatment as covariates. To assist in the planning of future randomized trials, we then calculated the standardized effect sizes of the intervention. Standardized effect sizes at each timepoint were calculated by dividing the mean differences of the changes (from baseline) by the pooled standard deviation (SD) of the changes for each outcome ${ }^{29}$.

Standardized effect size $=[$ mean $(\Delta$ intervention $)-\operatorname{mean}(\Delta$ control $)] /$ SD (pooled $\Delta$ intervention and $\Delta$ control)

Additionally, to evaluate the slope of change over the entire time span of the study in both groups, we used models assessing the disease outcomes at $3,6,9$, and 21 months, using time and group as categorical factors and interactions between time and group differences by the MIXED procedure in SAS version 9.2 (SAS Institute) for the 9-month and 21-month followup. RA medications were abstracted and tabulated for all participants at each timepoint of the trial. We ran the GENMOD procedure in SAS version 9.2 to analyze medication usage trends with time and group as categorical factors and interactions between time and group. We used the more conservative intention-to-treat (ITT) approach for analysis where participants who did not complete the followup period had their last value carried forward ${ }^{30}$. We also calculated mean treatment effects posttreatment and 21 months later in those who completed the protocol. To test the robustness of the ITT results, we ran a sensitivity analysis that included study completers and then an analysis that replaced the 9-month and 21-month followup values of those who dropped out with their baseline values. A 2-sided $\mathrm{p}$ value of $<0.05$ was considered statistically significant.

\section{RESULTS}

Figure 1, based on the Consolidated Standards of Reporting Trials, details the trial recruitment and followup. Informational letters were sent to 857 potential subjects, and they were asked to send back a postcard if they did not wish to be contacted $(n=209)$. Phone calls were made to 358 potential participants to determine interest and eligibility; 259 declined participation and 290 were not contacted. Twenty potential participants who agreed to participate did not meet inclusion criteria, and 79 were randomized to study groups. The time commitment was the biggest reason for declining participation.

An analysis of participants $(n=79)$ versus those who declined participation $(n=468)$ revealed that participants 


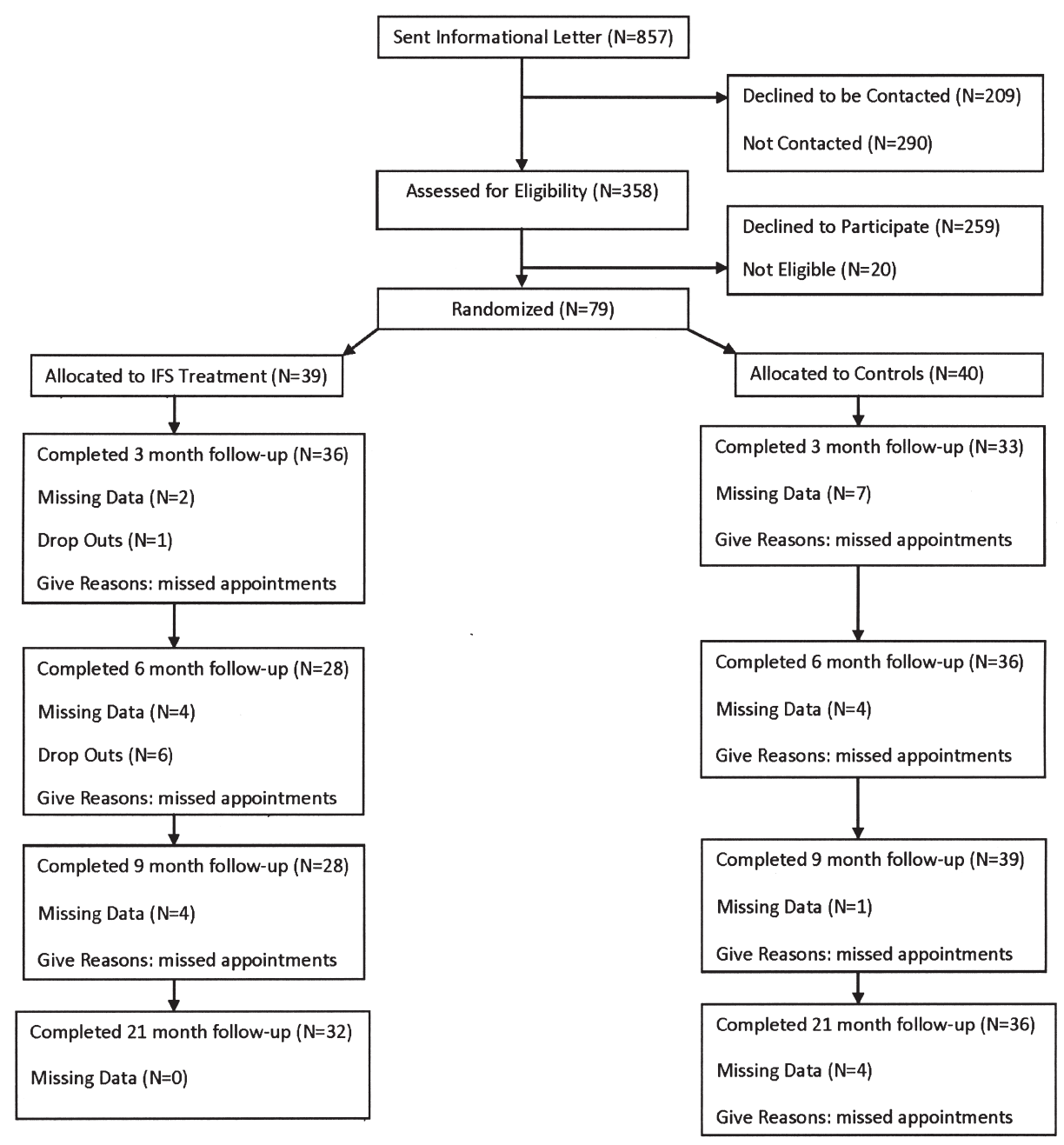

Figure 1. Flow chart of the Internal Family Systems (IFS) intervention program.

were more likely to be younger [mean, yrs (SD), 58.1 (12.8) vs $61.9(12.7), \mathrm{p}=0.02$ ], female [mean \% (n), 89 (30) vs 80 (39), $\mathrm{p}=0.04]$, and were more likely to be taking anti-tumor necrosis factor (TNF) inhibitor therapy [mean \% (n) 64 (48) vs $42(49), p=0.0003$ ]. Participants were not more likely to be married, or have a longer disease duration compared to nonparticipants. The dropouts and missed appointments at each followup are detailed in Figure 1.

Baseline characteristics. Table 2 details the baseline data for 79 participants by group. The mean age was $58 \mathrm{yrs}, 90 \%$ were female, and $93 \%$ were white. More than half the subjects were married. There was similar RA medication usage between groups, except that the IFS group had a higher prevalence of NSAID usage $(35 \%$ vs $12.5 \%, \mathrm{p}=$ 0.02 ). The group as a whole had anxiety levels that were similar to the norms of college students. The study participants suffered from minimal depression, on average. Baseline characteristics were balanced between groups on all the baseline outcome variables, except that the IFS group had higher disease activity and lower self-efficacy than the education group.

Feasibility of the intervention. Ninety-seven percent of the IFS group participants completed the 3-month followup. Seven subjects dropped out of the IFS treatment arm by 6 months and 32 had completed the protocol (82\%). The reasons for dropping out were difficulties with travel to meetings $(n=1)$, the time requirement involved in the study $(\mathrm{n}=2)$, limitations due to other illnesses $(\mathrm{n}=2$; a new diagnosis of cancer and a revision shoulder arthroplasty), and a perception of adequate benefit from the intervention after 3 months $(n=2)$. There were no differences in the baseline clinical, demographic, or outcome values between study completers versus those who dropped out (data not shown). There were 12 IFS group meetings throughout the study and all study subjects attended on average 7.4 meetings; $79 \%$ attended at least half of the group sessions. Study completers attended on average 8.4 group meetings. All subjects were able to actively participate in the IFS 
Table 2. Baseline characteristics of study participants.

\begin{tabular}{|c|c|c|c|}
\hline IFS & $\begin{array}{l}\text { Treatment Group, } \\
\quad \mathrm{n}=39\end{array}$ & $\begin{array}{l}\text { Controls, } \\
\mathrm{n}=40\end{array}$ & $\mathrm{p}$ \\
\hline Age, yrs; mean (SD) & $57.8(13.8)$ & $58.5(12.0)$ & 0.82 \\
\hline Female, n (\%) & $36(92.3)$ & $35(87.5)$ & 0.48 \\
\hline White, n (\%) & $35(89.7)$ & $38(97.4)$ & 0.17 \\
\hline Married, n (\%) & $21(53.9)$ & $28(70.0)$ & 0.14 \\
\hline Income $>70 \mathrm{~K} ; \mathrm{n}(\%)^{*}$ & $18(51.4)$ & $18(56.3)$ & 0.76 \\
\hline Disease duration, yrs; mean (SD) $)^{\dagger}$ & $18.9(10.8)$ & $13.9(9.2)$ & 0.06 \\
\hline \multicolumn{4}{|l|}{ Medications, $\mathrm{n}(\%)^{\ddagger}$} \\
\hline Analgesics & $5(13.5)$ & $4(10.0)$ & 0.63 \\
\hline NSAID & $13(35.1)$ & $5(12.5)$ & 0.02 \\
\hline Corticosteroids & $8(21.6)$ & $13(32.5)$ & 0.28 \\
\hline DMARD (nonbiological) & $26(70.3)$ & $28(70.0)$ & 0.98 \\
\hline Biological therapy & $13(35.1)$ & $16(40.0)$ & 0.66 \\
\hline \multicolumn{4}{|l|}{ Disease activity variables, mean (SD) } \\
\hline DAS28-CRP4 & $3.8(1.4)$ & $3.0(1.2)$ & 0.01 \\
\hline RADAI joint score & $1.9(1.5)$ & $1.5(1.3)$ & 0.19 \\
\hline \multicolumn{4}{|l|}{ Psychological variables, mean (SD) } \\
\hline Beck Depression Inventory & $10.2(7.0)$ & $9.2(7.4)$ & 0.55 \\
\hline Spielberger State Trait Anxiety Index & $\mathrm{x} \quad 38.4(8.9)$ & $36.5(10.8)$ & 0.42 \\
\hline Self-compassion scale & $20.9(4.1)$ & $22.5(4.5)$ & 0.10 \\
\hline Lorig Self Efficacy & $68.2(16.2)$ & $77.1(16.0)$ & 0.02 \\
\hline \multicolumn{4}{|l|}{ Functional variables, mean (SD) } \\
\hline SF-12 physical function & $51.9(32.6)$ & $64.1(32.8)$ & 0.11 \\
\hline \multicolumn{4}{|l|}{ Visual analog scale } \\
\hline Pain & $43.2(28.0)$ & $31.7(27.2)$ & 0.07 \\
\hline Sleep & $1.0(0.9)$ & $0.8(0.9)$ & 0.32 \\
\hline
\end{tabular}

* Data missing on 8 controls and 4 IFS participants; ${ }^{\dagger}$ Deleting 1 outlier; $\stackrel{\ddagger}{\ddagger}$ Data missing on 2 IFS participants. IFS: Internal Family Systems; NSAID: nonsteroidal antiinflammatory drugs; DMARD: disease-modifying antirheumatic drug; DAS28-CRP4: Disease Activity Score-28-C-reactive Protein 4; RADAI: Rheumatoid Arthritis Disease Activity Index; SF-12: Short Form-12.

protocol. Seven subjects in the education group missed their 3-month followup, 4 missed their 6-month followup, 1 missed the 9-month, and 4 missed their final evaluations. However, no education group participants requested that the study stop mailing them educational information (Figure 1).

Effect of the intervention. Table 3 shows the baseline and outcome data for each group at the 9-month and 21-month followup visits. Treatment effects and effect sizes were calculated posttreatment and 1 year later.

Posttreatment effects. At the end of the 9-month intervention, self-assessed joint pain, physical function, self-compassion, and overall pain treatment effects favored the IFS group compared with those receiving education alone [mean treatment effects (SD), RADAI joint score -0.9 (1.4), $\mathrm{p}=0.01 ; \mathrm{SF}-12 \mathrm{pf}$ score 14.6 (25.3), $\mathrm{p}=0.02$; VAS pain $-14.9(29.1), p=0.04$; Neff score $1.6(3), p=0.03]$. IFS participants showed a greater but nonsignificant mean improvement in anxiety [STAI $-2.2(10.2), \mathrm{p}=0.39$ ], depressive symptoms [BDI $-2.9(6.6), \mathrm{p}=0.07]$, and sleep quality $[-0.3(0.7), \mathrm{p}=0.11]$ at the end of the intervention compared with the education group. Among individuals who completed the protocol, significant treatment effects favoring the IFS group were found in depressive symptoms and self-efficacy at 9 months $[\mathrm{BDI}-4(6.8), \mathrm{p}=0.03$; Lorig Arthritis Self Efficacy score 13 (16.5), $\mathrm{p}=0.03$ ].

Followup at 21 months. One year after the intervention concluded, the IFS group had sustained improvements in self-assessed joint pain [mean treatment effects (SD), -0.6 (1.1), $\mathrm{p}=0.04)$ ], self-compassion [1.8 (2.8), $\mathrm{p}=0.01]$, and depressive symptoms $[-3.2(5.0), \mathrm{p}=0.01]$. There were no sustained improvements in anxiety, self-efficacy, or disease activity. Among subjects who completed the 21-month followup, outcomes were not different from the ITT analysis findings.

Sensitivity analysis. To evaluate the robustness of the ITT analyses, we ran a sensitivity analysis at both the posttreatment and 21-month followup visits. We ran 1 analysis with completers alone and 1 that evaluated the outcomes at the 9-month and 21-month timepoints by substituting the dropout participants' scores with their baseline values. Substituting baseline values for the dropouts revealed that for the 9-month analysis there was no longer a significant treatment effect in pain [mean treatment effect (SD), -12.9 (28.5), $\mathrm{p}=0.06$ ]; however, the IFS group showed greater improvement in self-efficacy [10.8 (15.9), $\mathrm{p}=0.05]$. The 21 -month analysis was no different from the 
Table 3. Mean $(95 \% \mathrm{CI})$ scores, treatment effects (between group change at 9 and 21 months), and p values estimated using t tests on the differences between the Internal Family System (IFS) and education groups.

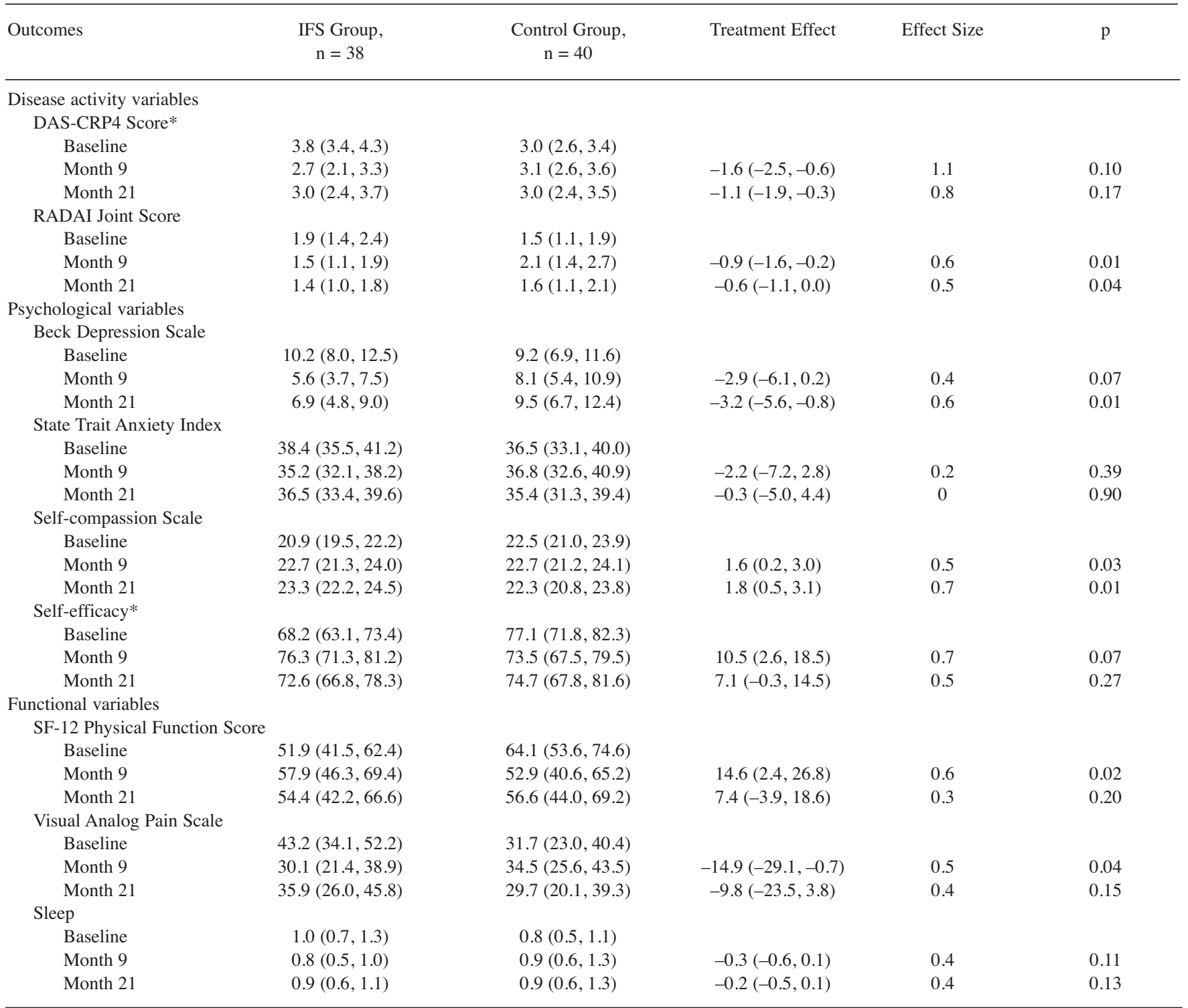

* Adjusting for baseline differences in outcome score using general linear regression. Measure difference = intercept + group + baseline measure. DAS-CRP4: Disease Activity Score 28-C-reactive Protein 4; RADAI: Rheumatoid Arthritis Disease Activity Index; SF-12: Short form-12.

ITT analysis when substituting baseline values for the dropouts.

Group trends over time. Figure 2 demonstrates an overview of the trend during the entire 21-month time span of the study using a repeated measures analysis. Baseline scores and responses at the 3-, 6-, 9-, and 21-month followup visits in the 2 groups showed a slope of change favoring treatment 1 year later in all outcomes, except for anxiety level, physical function, and pain. At the 9-month posttreatment timepoint, all outcomes trended toward significant improvement in the IFS group compared with the education group, except for anxiety.
Medication usage. There was no statistically different change in patterns of arthritis medication use over time in the IFS group compared with the education group at the 9-month posttreatment evaluation and 21 months later.

\section{DISCUSSION}

In this proof-of-concept trial, a 36-week IFS-based intervention was safe and well accepted by subjects enrolled in the program; $82 \%$ of the IFS intervention group completed the protocol and no adverse effects were reported. One year after the intervention ended, self-assessed joint pain, self-compassion, and depressive symptoms remained 

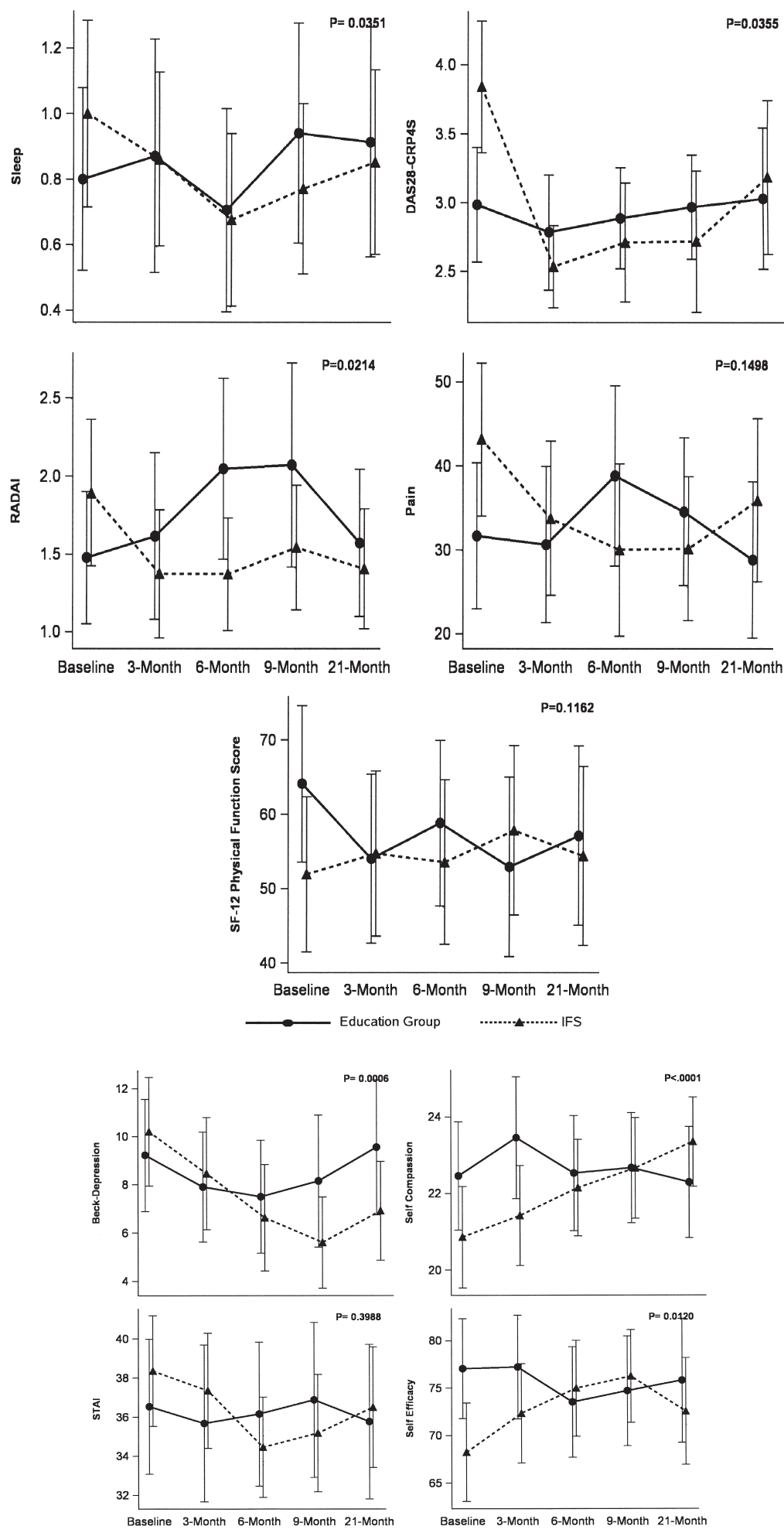

Figure 2. Mean differences with 95\% CI between the Internal Family Systems (IFS) intervention group and the education group in study outcomes at baseline and 3,6, 9, and 21 months. P values reflect mixed model repeated measures analyses. DAS28-CRP4S: Disease Activity Score-28-C-reactive Protein 4; RADAI: Rheumatoid Arthritis Disease Activity Index; SF-12: Short Form-12; STAI: State Trait Anxiety Inventory. 
improved. The improvement in overall pain and function that occurred at the end of the intervention was not sustained 1 year later and there was no significant improvement in anxiety throughout the study. Our study suggests that the IFS model may provide some sustainable benefits to patients with RA.

Our results are consistent with other psychotherapeutic interventions that demonstrate improvement in RA disability, pain, depression, and/or coping efficacy $5,6,7,31,32$. Reductions in disease activity and joint inflammation have been reported after cognitive behavioral therapy (CBT), particularly in those subjects with earlier-onset RA $^{4,9,10,11,14,32}$. Several recent reports suggest that interventions teaching emotional regulation, such as writing about stressful experiences or using mindfulness meditation, have resulted in less disease activity and joint inflammation in individuals with $\mathrm{RA}^{4,8}$. However, in 1 study, mindfulness meditation resulted in improvement of psychological distress and well-being, but did not result in improvement in DAS28 scores ${ }^{7}$. In another mindfulness study, improvement in overall well-being, self-efficacy, fatigue, and function occurred, but there was no improvement in disease activity and pain 1 year $\operatorname{later}^{18}$. A prior metaanalysis, including studies of CBT and the relaxation response, reported that in those subjects with a shorter duration of RA, certain psychotherapeutic interventions had a moderate effect on joint inflammation ${ }^{13}$. There was a similar decline in self-assessed joint pain among subjects in our study, and they had a longer disease duration (on average 16 yrs).

While the IFS intervention was tailored toward reducing disease activity and joint pain, it also produced a sustainable improvement in depressive symptoms despite baseline Beck scores indicating only mild symptoms. Self-compassion and self-efficacy (among study completers) also improved more in the IFS group and self-compassion improvements persisted 1 year later. While there was a trend toward reduced anxiety, no significant improvements occurred 1 year later, perhaps because of participants' baseline STAI scores indicating minimal anxiety. Further studies of the IFS intervention's effect on subjects with higher levels of anxiety would be informative.

The IFS intervention could have affected RA symptoms in a number of ways. Skeletal muscle relaxation can reduce joint pain. Stress has been linked to exacerbations in RA activity, and catastrophizing, in particular, affects many pain-related outcomes in RA and other rheumatic diseases $33,34,35,36$. A central goal of the IFS approach is to enhance self-compassion; by doing so, psychological reactions to pain, hopelessness, and anxiety may improve, as may the degree of inner conflict, perceived stress, and its effect on physical symptoms. Work on self-compassion by $\mathrm{Neff}^{28}$ shows promise in improving physical health. As an example, Adams and Leary ${ }^{37}$ demonstrated that improving self-compassion can reduce maladaptive overeating. In our current study, self-compassion began improving by 6 months, until the intervention end. It tracked along with the improvement in depressive symptoms and pain. Self-compassion may be an important mediating variable in the study participants' improvements.

Despite its name, IFS is primarily an individual psychotherapeutic modality and not a family systems technique. It teaches the skills of attending to an individual's internal experience, but it is different from cognitive behavior therapies, which primarily focus on restructuring distorted thinking or unrealistic cognitive appraisals to achieve changes in behavior or emotion ${ }^{38,39}$. IFS combines elements of other psychotherapies to help individuals learn about and change the beliefs and emotions that surround their symptoms. First, similar to mindfulness-based meditation practices and psychotherapies ${ }^{40,41}$, individuals are helped to separate from their thoughts and emotions, and access a mindful state called "Self" in IFS. Rather than simply mindfully observing their internal processes, however, they are encouraged to engage in a practice of inner inquiry, asking questions of those thoughts and emotions. Through this practice, individuals often access memories of attachment injuries or traumas that are then witnessed by the individuals while in the mindful state of Self. This is similar to the technique of exposure therapy ${ }^{42}$, except that in IFS the individual brings up those memories while in a specific state of compassion, calm, and clarity (Self) and therefore is able to process or update those trauma-based emotions and beliefs rapidly $43,44,45$.

Our study had several limitations. While $82 \%$ of the IFS group participants completed the program, most of those who declined did so because of the 9-month time commitment. We sent out an unsolicited informational letter to all of the patients with RA enrolled in our BRASS registry cohort. Many of these individuals may not have been interested in such a long self-management program. Other similar trials that initially sent out an information letter or recruited widely through advertisement have had overall yields similar to our study ${ }^{7,18}$. However, it is a limitation of the program that the time commitment was 36 weeks. Future efficacy studies should consider crafting a shorter program, which may increase acceptability. Additionally, we did not administer formal questionnaires assessing acceptability, satisfaction, or face validity, which limits our ability to comment specifically on our study's feasibility.

While our education group received contact and attention, the amount of attention was less intense than in our treatment group (1-h meeting and mailed educational materials vs 15 individual sessions and 12 group meetings). Differences in study contact, counseling, and group contact, independent of the type of intervention, could have accounted for the positive treatment effects. Further, the intervention group may have had an enhanced belief in the

Personal non-commercial use only. The Journal of Rheumatology Copyright (c) 2013. All rights reserved. 
treatment credibility and efficacy of the IFS intervention compared with those in the education group and this could also have contributed to the differential improvement. Additionally, there was greater attrition in the IFS group compared with the educational group, which may also have affected the study results. The education group received mailings of literature and followup phone calls, while the intervention group had to travel to meetings and groups. No member of the education group asked us to stop mailing them educational literature about RA. The single most common reason for dropping out of the study in the IFS group was difficulty with travel to meetings and the conflict it posed with other commitments. While there were no differences in baseline clinical, demographic, and outcome variables between study completers and dropouts, this differential attrition lends uncertainty in interpreting any positive results. We ran several alternate analyses to evaluate the robustness of our ITT findings, including a sensitivity analysis that included completers, and outcome variables with baseline value substitutions in the subjects who didn't finish the program. Results from the sensitivity analyses did not vary widely from our ITT results.

All of the therapists provided written documentation of the salient contents of each meeting and the elements of the IFS treatment; however, it is a limitation of the study that the sessions were not audiotaped. This would have helped evaluate the intervention's fidelity and possibly identify the program's most influential exercises. Future studies of an IFS program should include audiotaping of sessions. Additionally, exercise and physical activity, which may be a mediating variable of mood and functional outcomes in the study, was not specifically measured. The SF-12 physical function subscale does assess the capacity for some basic physical activities, but it does not measure exercise capacity or endurance. Exercise affects mood and disease activity and it may have differentially affected how each participant responded to the intervention.

Two other limitations in our study deserve separate comment. Despite randomization, the IFS group had a higher disease activity score and lower self-efficacy at baseline than the education group. Improvements in disease activity and self-efficacy could be attributable to regression toward the mean. To account for this effect, we adjusted the outcomes for the baseline differences in these 2 variables and also included a sensitivity analysis at the 9-month and 21-month timepoints to evaluate the robustness of the group trends over time. Second, our study was powered to detect an effect size of 0.70 , an estimate drawn from prior studies of cognitive behavioral interventions with patients with RA who, at the time, did not have access to anti-TNF and other biologic therapies ${ }^{5,11}$. These studies likely included patients with more active disease who had greater potential for improvement. About half of our subjects were receiving anti-TNF therapies; with less active disease we would be unable to detect a statistically significant effect in disease activity (and other outcomes) that was $<0.70$.

The IFS intervention took 36 weeks, and is longer than many prior behavioral and self-management interventions. While these shorter interventions can be effective, they have had variable sustained benefits. We wanted to evaluate the sustainability of a 9-month intervention, hoping that some of the program's skills would be incorporated into the subjects' daily lives. Sustainable improvements may be beneficial to patients with a chronic illness such as RA. As an example, in a followup study of recurrent cardiovascular events in patients with coronary heart disease, an initial year of cognitive behavioral therapy reduced rates of fatal and nonfatal recurrent cardiovascular events and lowered all-cause mortality over the ensuing 8 years of followup ${ }^{46}$. There was a strong dose-response effect between intervention attendance and benefit; overall, the intervention's efficacy was equal to or stronger than many secondary drug prevention trials ${ }^{47}$. In our current study, there was a sustained improvement in depressive symptoms 1 year after the intervention's end. Depression in RA has been linked to an increased risk for other comorbidities and an elevated mortality ${ }^{48,49}$. Nicassio, et $a l^{50,51}$, has demonstrated the complex interplay of increased pain perception, sleep disturbance, helplessness, and depression in RA. Given the frequent co-occurrence of depression among patients with RA, an intervention with sustainable benefit in depressive symptoms may produce sustainable health benefits in the long term.

An IFS intervention tailored to work with patients with RA appears feasible and acceptable and may complement medical management of the disease. The sustained improvement in depressive symptoms, self-compassion, and reduction in self-reported joint pain suggests that the program participants may have incorporated some of the program's strategies into their daily lives. Integrating an IFS-based self-management program that incorporates active mindfulness into an RA clinic may be particularly helpful to patients. Future efficacy studies are warranted, in particular those that compare the IFS technique with other psychotherapeutic interventions.

\section{ACKNOWLEDGMENT}

We are grateful for the participation and help of the BRASS volunteers and the R.B. Brigham Arthritis Center rheumatologists: Susan Ritter, MD, PhD; Erika Noss, MD, PhD; and Yvonne Lee, MD, MSc. We thank Paul Neustadt, MSW, and Deborah Bloch, $\mathrm{PhD}$, for their contributions and Dorothy Wheeler, $\mathrm{PhD}$, for her guidance and inspiration to conduct the intervention.

\section{REFERENCES}

1. Katz PP, Yelin EH. Life activities of persons with rheumatoid arthritis with and without depressive symptoms. Arthritis Care Res 1994;7:69-77.

2. Katz PP. The impact of rheumatoid arthritis on life activities. Arthritis Care Res 1995;8:272-8. 
3. Dickens C, Creed F. The burden of depression in patients with rheumatoid arthritis. Rheumatology 2001;40:1327-30.

4. Zautra AJ, Davis MC, Reich JW, Nicassario P, Tennen H, Finan P, et al. Comparison of cognitive behavioral and mindfulness meditation interventions on adaptation to rheumatoid arthritis for patients with and without history of recurrent depression. J Consult Clin Psychol 2008;76:408-21.

5. Bradley LA, Young LD, Anderson KO, Turner RA, Agudelo CA, McDaniel L, et al. Effects of psychological therapy on pain behavior of rheumatoid arthritis patients. Treatment outcome and six-month followup. Arthritis Rheum 1987;30:1105-14.

6. Evers AW, Kraaimaat FW, van Riel PL, de Jong AJ. Tailored cognitive-behavioral therapy in early rheumatoid arthritis for patients at risk: a randomized controlled trial. Pain 2002;100:141-53.

7. Pradhan EK, Baumgarten M, Langenberg P, Handwerger B, Gilpin AK, Magyari T, et al. Effect of mindfulness-based stress reduction in rheumatoid arthritis patients. Arthritis Rheum 2007;57:1134-42.

8. Smyth JM, Stone AA, Hurewitz A, Kaell A. Effects of writing about stressful experiences on symptom reduction in patients with asthma or rheumatoid arthritis: a randomized trial. JAMA 1999;281:1304-9.

9. Sharpe L, Sensky T, Timberlake N, Ryan B, Brewin CR, Allard S, et al. A blind, randomized, controlled trial of cognitive-behavioural intervention for patients with recent onset rheumatoid arthritis: preventing psychological and physical morbidity. Pain 2001;89:275-83.

10. Sharpe L, Sensky T, Timberlake N, Ryan B, Allard S. Long-term efficacy of a cognitive behavioural treatment from a randomized controlled trial for patients recently diagnosed with rheumatoid arthritis. Rheumatology 2003;42:435-41.

11. O'Leary A, Shoor S, Lorig K, Holman HR. A cognitive-behavioral treatment for rheumatoid arthritis. Health Psychol 1988;7:527-44.

12. Dixon KE, Keefe FJ, Scipio CD, Perri LM, Abernethy AP. Psychological interventions for arthritis pain management in adults: a meta-analysis. Health Psychol 2007;26:241-50.

13. Astin JA, Beckner W, Soeke K, Hochberg MC, Berman B. Psychological interventions for rheumatoid arthritis: a meta-analysis of randomized controlled trials. Arthritis Rheum 2002;47:291-302.

14. Riemsma RP, Kirwan JR, Taal E, Rasker JJ. Patient education for adults with rheumatoid arthritis. Cochrane Database Syst Rev 2003;2:CD003688.

15. Schwartz R. Internal Family Systems therapy. New York: Guilford Press; 1995.

16. Hamilton NA, Zautra AJ, Reich J. Individual differences in emotional processing and reactivity to pain among older women with rheumatoid arthritis. Clin J Pain 2007;23:165-72.

17. Zautra AJ, Yocum DC, Villanueva I, Smith B, Davis MC, Attrep J, et al. Immune activation and depression in women with rheumatoid arthritis. J Rheumatol 2004;31:457-63.

18. Zangi HA, Mowinckel P, Finset A, Eriksson LR, Høystad TØ, Lunde AK, et al. A mindfulness-based group intervention to reduce psychological distress and fatigue in patients with inflammatory rheumatic joint diseases: a randomised controlled trial. Ann Rheum Dis 2012;71:911-7.

19. Schwartz R. The Center for Self Leadership [unpublished data]. Oak Park, Illinois: 2013.

20. Iannaccone CK, Lee YC, Cui J, Frits ML, Glass RJ, Plenge RM, et al. Using genetic and clinical data to understand response to disease-modifying anti-rheumatic drug therapy: data from the Brigham and Women's Hospital Rheumatoid Arthritis Sequential Study. Rheumatology 2011;50:40-6.

21. Stucki G, Liang MH, Stucki S, Brühlmann P, Michel BA. A self-administered rheumatoid arthritis disease activity index (RADAI) for epidemiologic research. Psychometric properties and correlation with parameters of disease activity. Arthritis Rheum 1995;38:795-8.

22. Fransen J, Häuselmann H, Michel BA, Caravatti M, Stucki G. Responsiveness of the self-assessed rheumatoid arthritis disease activity index to a flare of disease activity. Arthritis Rheum 2001;44:53-60.

23. Beck AT, Ward CH, Mendelson M, Mock J, Erbaugh J. An inventory for measuring depression. Arch Gen Psychiatry 1961;4:561-71.

24. Spielberger CD, Vagg PR. Psychometric properties of the STAI: a reply to Ramanaiah, Franzen, and Schill. J Pers Assess 1984; 48:95-7.

25. Ware J Jr, Kosinski M, Keller SD. A 12-Item Short-Form Health Survey: construction of scales and preliminary tests of reliability and validity. Med Care 1996;34:220-33.

26. Pincus T, Swearingen C, Wolfe F. Toward a multidimensional Health Assessment Questionnaire (MDHAQ): assessment of advanced activities of daily living and psychological status in the patient-friendly health assessment questionnaire format. Arthritis Rheum 1999;42:2220-30.

27. Lorig K, Chastain RL, Ung E, Shoor S, Holman HR. Development and evaluation of a scale to measure perceived self-efficacy in people with arthritis. Arthritis Rheum 1989;32:37-44.

28. Neff K. Development and validation of a scale to measure self compassion. Self Identity 2003;2:223-50.

29. Dworkin RH, Peirce-Sandner S, Turk DC, McDermott MP, Gibofsky A, Simon LS, et al. Outcome measures in placebo-controlled trials of osteoarthritis: responsiveness to treatment effects in the REPORT database. Osteoarthritis Cartilage 2011;19:483-92.

30. Altman DG, Schulz KF, Moher D, Egger M, Davidoff F, Elbourne $\mathrm{D}$, et al. The revised CONSORT statement for reporting randomized trials: explanation and elaboration. Ann Intern Med 2001;134:663-94.

31. Parker JC, Smarr KL, Buckelew SP, Stucky-Ropp RC, Hewett JE, Johnson JC, et al. Effects of stress management on clinical outcomes in rheumatoid arthritis. Arthritis Rheum 1995;38:1807-18.

32. Leibing E, Pfingsten M, Bartmann U, Rueger U, Schuessler G. Cognitive-behavioral treatment in unselected rheumatoid arthritis outpatients. Clin J Pain 1999;15:58-66.

33. van Lankveld W, van Helmond T, Näring G, de Rooij DJ, van den Hoogen F. Partner participation in cognitive-behavioral self-management group treatment for patients with rheumatoid arthritis. J Rheumatol 2004;31:1738-45.

34. Potter PT, Zautra AJ. Stressful life events' effects on rheumatoid arthritis disease activity. J Consult Clin Psychol 1997;65:319-23.

35. Harrington L, Affleck G, Urrows S, Tennen H, Higgins P, Zautra A, et al. Temporal covariation of soluble interleukin-2 receptor levels, daily stress, and disease activity in rheumatoid arthritis. Arthritis Rheum 1993;36:199-203.

36. Edwards RR, Bingham CO 3rd, Bathon J, Haythornthwaite JA. Catastrophizing and pain in arthritis, fibromyalgia, and other rheumatic diseases. Arthritis Rheum 2006;55:325-32.

37. Adams CE, Leary MR. Promoting self-compassionate attitudes toward eating among restrictive and guilty eaters. J Soc Clin Psychol 2007;26:1120-44.

38. Beck A. Cognitive therapy: nature and relation to behavior therapy. Behavior Therapy 1970;1:184-200.

39. Dobson K, ed. Handbook of cognitive-behavioral therapies, 3rd edition. New York: Guilford Press; 2009.

40. Kabat-Zinn J. An outpatient program in behavioral medicine for chronic pain patients based on the practice of mindfulness meditation: theoretical considerations and preliminary results. Gen Hosp Psychiatry 1982;4:33-47.

41. Evans S, Ferrando S, Findler M, Stowell C, Smart C, Haglin D. 
Mindfulness-based cognitive therapy for generalized anxiety disorder. J Anxiety Disord 2008;22:716-21.

42. Huppert JD, Roth DA, Foa EB. Cognitive-behavioral treatment of social phobia: new advances. Curr Psychiatry Rep 2003;5:289-96.

43. Sweezy M. The teenager's confession: regulating shame in internal family systems therapy. Am J Psychother 2011;65:179-88.

44. Sweezy M. Treating trauma after dialectical behavior therapy. J Psychother Integr 2011;21:90-102.

45. Twombly JH, Schwartz RC. The integration of the Internal Family Systems model and EMDR. In: Forgash C, Copeley M, eds. Healing the heart of trauma and dissociation with EMDR and ego state therapy. New York: Springer; 2008:295-311.

46. Gulliksson M, Burell G, Vessby B, Lundin L, Toss H, Svärdsudd K. Randomized controlled trial of cognitive behavioral therapy vs standard treatment to prevent recurrent cardiovascular events in patients with coronary heart disease: Secondary Prevention in Uppsala Primary Health Care project (SUPRIM). Arch Intern Med 2010;171:134-40.
47. Miller DB. Secondary prevention for ischemic heart disease. Relative numbers needed to treat with different therapies. Arch Intern Med 1997;157:2045-52.

48. Ang DC, Choi H, Kroenke K, Wolfe F. Comorbid depression is an independent risk factor for mortality in patients with rheumatoid arthritis. J Rheumatol 2005;32:1013-9.

49. Scherrer JF, Virgo KS, Zeringue A, Bucholz KK, Jacob T, Johnson $\mathrm{RG}$, et al. Depression increases risk of incident myocardial infarction among Veterans Administration patients with rheumatoid arthritis. Gen Hosp Psychiatry 2009;31:353-9.

50. Nicassio PM, Ormseth SR, Kay M, Custodio M, Irwin MR, Olmstead R, et al. The contribution of pain and depression to self-reported sleep disturbance in patients with rheumatoid arthritis. Pain 2012;153:107-12.

51. Nicassio PM, Wallston KA, Callahan LF, Herbert M, Pincus T. The measurement of helplessness in rheumatoid arthritis. The development of the arthritis helplessness index. J Rheumatol 1985;12:462-7. 